\title{
COMMUTATORS AS POWERS IN FREE PRODUCTS OF GROUPS
}

\author{
LEO P. COMERFORD, JR., CHARLES C. EDMUNDS, AND GERHARD ROSENBERGER
}

(Communicated by Ronald M. Solomon)

\begin{abstract}
The ways in which a nontrivial commutator can be a proper power in a free product of groups are identified.
\end{abstract}

It is well known that in a free group, a nontrivial commutator cannot be a proper power. This seems to have been noted first by Schützenberger [2]. It is, however, possible for a nontrivial commutator to be a proper power in a free product. Our aim in this paper is to determine the ways in which this can happen.

Theorem 1. Let $G=*_{i \in I} G_{i}$, the free product of nontrivial free factors $G_{i}$. If $V, X, Y \in G$ and $V^{m}=X^{-1} Y^{-1} X Y=[X, Y]$ for some $m \geq 2$, then either

(1.1) $V \in W^{-1} G_{i} W$ for some $W \in G, i \in I$, and $V^{m}$ is a commutator in $W^{-1} G_{i} W ;$ or

(1.2) $m$ is even, $V=A B$ with $A^{2}=B^{2}=1$, and $V^{m}=\left[A, B(A B)^{(m-2) / 2}\right]$; or

(1.3) $m$ is odd, $V=A C^{-1} A C$ with $A^{2}=1$, and $V^{m}=[A$, $\left.C\left(A C^{-1} A C\right)^{(m-1) / 2}\right]$; or

(1.4) $m=6, V=A B$ with $A^{2}=B^{3}=1$, and $V^{6}=\left[B^{-1} A B A, B(A B)^{2}\right]$; or

(1.5) $m=3, V=A B$ with $A^{3}=B^{3}=1$, and $V^{3}=\left[B A^{-1}, B A B\right]$; or

(1.6) $m=2, V=A B$ with $A^{2}=1$ and $B^{-1}=C^{-1} B C$ for some $C \in G$, and $V^{2}=\left[C^{-1} A, B\right] ;$ or

(1.7) $m=4, V^{2}=A B C$ with $A^{2}=B^{2}=C^{2}=1$, and $V^{4}=[B A, B C]$.

We recall that in a free product every element of finite order lies in a conjugate of a free factor. Thus we have the following consequence of Theorem 1.

Corollary 2. Let $G=*_{i \in I} G_{i}$, where no $G_{i}$ has elements of even urder. If $V, X, Y \in G$ and $V^{m}=[X, Y]$ for some $m \geq 2$, then either $V \in W^{-1} G_{i} W$ for some $W \in G, i \in I$, and $V^{m}$ is a commutator in $W^{-1} G_{i} W$ or $m=3$, $V=A B$ for some $A, B \in G$ with $A^{3}=B^{3}=1$, and $V^{3}=\left[B A^{2}, B A B\right]$.

Part (1.7) of Theorem 1 is somewhat unsatisfactory in that it describes the form of $V^{2}$ rather than that of $V$. Among the ways in which an element $V$ of a free product may have $V^{2}=A B C$ with $A^{2}=B^{2}=C^{2}=1$ is $V=D E$

Received by the editors December 6, 1992.

1991 Mathematics Subject Classification. Primary 20E06; Secondary 20F12. 
with $D^{2}=E^{4}=1$, in which case $V^{2}=(D)\left(E^{2}\right)\left(E^{-1} D E\right)$. Not every solution is of this form, as shown by $G=\left\langle a, b ; a^{2}=b^{2}=(a b)^{2}=1\right\rangle *\left\langle c ; c^{2}=\right.$ 1) and $V=a c b c a b c$; here $V^{2}=(a c b c a)(b c a c b)(c a b c)$, a product of three elements of order two, but $V$ is not a product of two elements of finite order. A classification of elements $V$ satisfying the conditions of (1.7) has eluded us.

Relative to (1.6), we record the following well-known consequence of the Conjugacy Theorem for Free Products [1, Theorem IV.1.4].

Lemma 3. If $B$ is an element of a free product $G=*_{i \in I} G_{i}$ and $B^{-1}=C^{-1} B C$ for some $C \in G$, then either

(3.1) $B \in W^{-1} G_{i} W$ for some $W \in G, i \in I$, and there is a $C \in W^{-1} G_{i} W$ such that $B^{-1}=C^{-1} B C$ or

(3.2) $B=D E$ for some $D, E \in G$ with $D^{2}=E^{2}=1$.

Before proceeding with a proof of the theorem, we establish some notation and terminology for the free product $G=*_{i \in I} G_{i}$. Our usage is that of Lyndon and Schupp [1] unless otherwise noted. A product $P Q$ of elements $P$ and $Q$ of $G$ is reduced if one of $P, Q$ is trivial or if the last letter of the normal form of $P$ is not inverse to the first letter of the normal form of $Q$. The product $P Q$ is fully reduced if $P$ or $Q$ is trivial or if the last letter of the normal form of $P$ is from a free factor different from that of the first letter of the normal form of $Q$; we sometimes denote this by writing $P \cdot Q$. These notions extend to products of more than two factors, with the understanding that the noncancellation conditions continue to apply after trivial factors have been deleted. Thus a product $P_{1} \cdots P_{k}$ is fully reduced if and only if $\left|P_{1} \cdots P_{k}\right|=$ $\sum_{i=1}^{k}\left|P_{i}\right|$, where || denotes free product length.

An element $P$ of $G$ is cyclically reduced if $|P| \leq 1$ or the first and last letters of its normal form are not inverses and is fully cyclically reduced if $|P| \leq 1$ or the first and last letters of its normal form lie in different free factors of $G$.

A key ingredient in our analysis will be the characterization by Wicks of the fully reduced forms of a commutator in a free product. The following is a restatement of Lemma 6 of [3].

Lemma 4 (Wicks). If $U \in G=*_{i \in I} G_{i}$ is a commutator, either $U \in W^{-1} G_{i} W$ for some $W \in G, i \in I$, and $U$ is a commutator in $W^{-1} G_{i} W$, or some fully cyclically reduced conjugate of $U$ has one of the following fully reduced forms:

(4.1) $X^{-1} a_{1} X a_{2}$ with $X \neq 1, a_{1} \neq 1, a_{1}, a_{2} \in G_{i}$ for some $i \in I$, and $a_{1}$ conjugate to $a_{2}^{-1}$ in $G_{i}$; or

(4.2) $X^{-1} a_{1} Y^{-1} a_{2} X a_{3} Y a_{4}$ with $X \neq 1, Y \neq 1, a_{1}, a_{2}, a_{3}, a_{4} \in G_{i}$ for some $i \in I$, and $a_{4} a_{3} a_{2} a_{1}=1$; or

(4.3) $X^{-1} a_{1} Y^{-1} b_{1} Z^{-1} a_{2} X b_{2} Y a_{3} Z b_{3}$ with $a_{1}, a_{2}, a_{3} \in G_{i}$ for some $i \in I$ and $a_{3} a_{2} a_{1}=1, b_{1}, b_{2}, b_{3} \in G_{j}$ for some $j \in I$ and $b_{3} b_{2} b_{1}=1$, and either not all of $a_{1}, a_{2}, a_{3}, b_{1}, b_{2}, b_{3}$ are in any one free factor of $G$ or each of $X, Y, Z$ is nontrivial.

As a final preliminary step, we examine the ways in which both an element and its inverse can occur as fully reduced subwords of a proper power in a free product. 
Lemma 5. Suppose that $V$ is a fully cyclically reduced element of $G=*_{i \in I} G_{i}$ with $|V| \geq 2$, that $m \geq 1$, and that, for some $X, R, S, T \in G, V^{m}=$ $X^{-1} \cdot R=S \cdot X \cdot T$. Then one of the following is true:

(5.1) $|X| \geq|V|, X=X_{1} \cdot B \cdot A$ and $V=A \cdot B$ for some $A, B, X_{1}$ with $A^{2}=B^{2}=1$, and $S X=V^{n} \cdot A$ for some $n<m$; or

$\frac{1}{2}|V|<|X|<|V|, X=X_{1} \cdot X_{2} \cdot X_{3}$ and $V=X_{3} \cdot X_{2}^{-1} \cdot X_{1} \cdot X_{2}$ for some $X_{1}, X_{2}, X_{3}$ with $X_{1}^{2}=X_{3}^{2}=1$, and $S=V^{n} \cdot X_{3} \cdot X_{2}^{-1}$ for some $n<m$; or

(5.3) $|X|<|V|, X=X_{1} \cdot X_{2}$ and $V=X_{2}^{-1} \cdot X_{1} \cdot X_{2} \cdot T_{1}$ for some $X_{1}, X_{2}, T_{1}$ with $X_{1}^{2}=1$, and $S=V^{n} \cdot X_{2}^{-1}$ for some $n<m$; or

(5.4) $|X|<|V|, X=X_{1} \cdot X_{2}$ and $V=X_{2} \cdot X_{1}^{-1} \cdot S_{2} \cdot X_{1}$ for some $X_{1}, X_{2}, S_{2}$ with $X_{2}^{2}=1$, and $S=V^{n} \cdot X_{2} \cdot X_{1}^{-1} \cdot S_{3}$ for some $n<m$; or

(5.5) $|X| \leq \frac{1}{2}|V|-1$ and $V=X^{-1} \cdot V_{2} \cdot X \cdot V_{3}$ for some nontrivial $V_{2}, V_{3}$ and $S=V^{n} \cdot X^{-1} \cdot V_{2}$ for some $n<m$.

Proof of Lemma 5. If $X$ is empty, clause (5.5) applies with $V=V_{2} \cdot V_{3}$ a fully reduced factorization of $V$ such that $S=V^{n} \cdot V_{2}$ for some $n<m$. We suppose, then, that $X \neq 1$.

If $|X| \geq|V|$, we factor $V$ as $A \cdot B$ so that $S X=V^{n} \cdot A$ with $|A|<|V|$. It follows that $X=X_{1} \cdot B \cdot A$ for some $X_{1}$. But since $X^{-1}=A^{-1} \cdot B^{-1} \cdot X_{1}^{-1}$ is an initial subword of $V^{m}=(A \cdot B)^{m}, A^{-1}=A$ and $B^{-1}=B$. This is the situation described in (5.1). We assume, henceforth, that $|X|<|V|$.

Let $n$ be the largest integer such that $\left|V^{n}\right| \leq|S|$, and let $S_{1}, V_{1}$ be such that $S=V^{n} \cdot S_{1}$ and $V=X^{-1} \cdot V_{1}$. We cannot have $\left|S_{1}\right|=|X|$ or $\left|S_{1}\right|+|X|=|V|$, for that would violate our hypotheses on the fully reduced factorizations of $V^{m}$.

Suppose that $\left|S_{1}\right|<|X|$ and $\left|S_{1}\right|+|X|>|V|$. Then $X$ factors as $X_{1} \cdot X_{2} \cdot X_{3}$ with $X^{-1}=S_{1} \cdot X_{1}^{-1}, V=S_{1} \cdot X_{1} \cdot X_{2}$, and $X_{1}$ and $X_{2}$ nonempty. Now $S_{1}=X_{3}^{-1} \cdot X_{2}^{-1}$, so $V=X_{3}^{-1} \cdot X_{2}^{-1} \cdot X_{1} \cdot X_{2}$. But $S X=V^{n+1} \cdot X_{3}$, which implies that $X_{3}^{-1}=X_{3}$, and $V=X_{3}^{-1} \cdot X_{2}^{-1} \cdot X_{1}^{-1} \cdot V_{1}$, which yields $X_{1}^{-1}=X_{1}$. This is the situation of (5.2), and we note that $|V|<\left|S_{1}\right|+|X|$ and $\left|S_{1}\right|<|X|$ imply that $|V|<2|X|$.

Next suppose that $\left|S_{1}\right|<|X|$ and $\left|S_{1}\right|+|X|<|V|$. Then $X$ factors as $X_{1} \cdot X_{2}$ with $S_{1}=X_{2}^{-1}$ and $V=S_{1} \cdot X \cdot T_{1}$ for some $T_{1}$, so $V=X_{2}^{-1} \cdot X_{1} \cdot$ $X_{2} \cdot T_{1}=X_{2}^{-1} \cdot X_{1}^{-1} \cdot V_{1}$. It follows that $X_{1}^{-1}=X_{1}$, and we are in situation (5.3).

Now suppose that $\left|S_{1}\right|>|X|$ and $\left|S_{1}\right|+|X|>|V|$. We factor $X$ as $X_{1} \cdot X_{2}$ with $V=S_{1} \cdot X_{1}$ and factor $S_{1}$ as $X^{-1} \cdot S_{3}$. Then $V=X_{2}^{-1} \cdot X_{1}^{-1} \cdot S_{3} \cdot X_{1}$ and, since $S \cdot X=V^{n+1} \cdot X_{2}, X_{2}^{-1}=X_{2}$; this is (5.4).

Finally, suppose that $\left|S_{1}\right|>|X|$ and $\left|S_{1}\right|+|X|<|V|$. In this case, $S_{1}$ factors as $X^{-1} \cdot V_{2}$ for some $V_{2}$ and $V=S_{1} \cdot X \cdot V_{3}$ for some $V_{3}$. Then $V=X^{-1} \cdot V_{2} \cdot X \cdot V_{3}$, where necessarily $V_{2}$ and $V_{3}$ are nonempty, and (5.5) applies.

Proof of Theorem 1. Each of the forms specified for $V$ (or, in (1.7), $V^{2}$ ) in the conclusion of Theorem 1 is preserved if $V$ is replaced by a conjugate of itself, so we lose no generality in assuming that $V$ is fully cyclically reduced. If $V \in G_{i}$ for some $i \in I$, then Lemma 4 tells us that (1.1) holds. We suppose, then, that $|V| \geq 2$. 
By Lemma 4, some fully cyclically reduced conjugate of $V^{m}$ has the form specified in (4.1), (4.2), or (4.3). After again replacing $V$ by a fully cyclically reduced conjugate and relabeling in (4.2) and (4.3) if necessary, we may assume that $V^{m}$ has form (4.1), or form (4.2) with $|X| \geq|Y|$, or form (4.3) with $|X| \geq|Y|$ and $|X| \geq|Z|$.

Let $P=a_{1}$ and $Q=a_{2}$ in form (4.1), $P=a_{1} Y^{-1} a_{2}$ and $Q=a_{3} Y a_{4}=$ $a_{3} Y a_{1}^{-1} a_{2}^{-1} a_{3}^{-1}$ in form (4.2), and $P=a_{1} Y^{-1} b_{1} Z^{-1} a_{2}$ and $Q=b_{2} Y a_{3} Z b_{3}=$ $b_{2} Y a_{1}^{-1} a_{2}^{-1} Z b_{1}^{-1} b_{2}^{-1}$ in form (4.3). In each instance, $V^{m}=X^{-1} \cdot P \cdot X \cdot Q$ and $Q$ is conjugate to $P^{-1}$ in $G$. Further, $|P|=|Q|=1$ in (4.1), $|P| \leq|X|+2$ and $|Q| \leq|X|+2$ in (4.2), and $|P| \leq 2|X|+3$ and $|Q| \leq 2|X|+3$ in (4.3). We proceed by cases according to which clause of the conclusion of Lemma 5 is satisfied, with $R=P X Q, S=X^{-1} P$, and $T=Q$.

Case (5.1). Suppose that $X=X_{1} \cdot B \cdot A$ and $V=A \cdot B$ for some $X_{1}, A, B$ with $A^{2}=B^{2}=1$, that $X_{1}^{-1} P X_{1}=(A B)^{k} A$ for some $k, 0 \leq k \leq m-3$, and that $Q=B(A B)^{m-k-3}$.

If $m$ is even, (1.2) is satisfied, while if $m$ is odd, $Q$ conjugate to $P^{-1}$ implies that $B$ is conjugate to $A$ and (1.3) holds.

Case (5.2). Suppose that $X=X_{1} \cdot X_{2} \cdot X_{3}$ and $V=X_{3} \cdot X_{2}^{-1} \cdot X_{1} \cdot X_{2}$ for some $X_{1}, X_{2}, X_{3}$ with $X_{1}^{2}=X_{3}^{2}=1$, that $P=X_{2} X_{3} X_{2}^{-1}\left(X_{1} X_{2} X_{3} X_{2}^{-1}\right)^{k}$ for some $k, 0 \leq k \leq m-3$, and $Q=X_{2}^{-1} X_{1} X_{2}\left(X_{3} X_{2}^{-1} X_{1} X_{2}\right)^{m-k-3}$.

As in the previous case, (1.2) applies if $m$ is even, and if $m$ is odd, $Q$ conjugate to $P^{-1}$ implies that $X_{3}$ is conjugate to $X_{1}$ and (1.3) obtains.

Case (5.3). Suppose that $|X|<|V|, X=X_{1} \cdot X_{2}$ and $V=X_{2}^{-1} \cdot X_{1} \cdot X_{2} \cdot T_{1}$ for some $X_{1}, X_{2}, T_{1}$ with $X_{1}^{2}=1$, and that $P=X_{2} T_{1} X_{2}^{-1}\left(X_{1} X_{2} T_{1} X_{2}^{-1}\right)^{k}$ for some $k, 0 \leq k \leq m-2$, and $Q=T_{1}\left(X_{2}^{-1} X_{1} X_{2} T_{1}\right)^{m-k-2}$.

We first notice that since $|P| \leq 2|X|+3 \leq 2|V|+1$ and $|Q| \leq 2|X|+3 \leq$ $2|V|+1$, we have $m \leq 6$. Now $Q$ is conjugate to $P^{-1}$, so $P$ and $Q$ must have fully cyclically reduced conjugates of the same length. It is not hard to see that this implies that either $k=m-k-2$ or $T_{1}^{2}=1$. If $T_{1}^{2}=1$, we find as in previous cases that (1.2) applies if $m$ is even and that (1.3) applies if $m$ is odd. We suppose, then, that $T_{1}^{2} \neq 1$ and $k=m-k-2$. The possibilities to consider are that $m=2$ and $k=0, m=4$ and $k=1$, and $m=6$ and $k=2$.

If $m=2$ and $k=0, T_{1}$ is conjugate to $T_{1}^{-1}$ and (1.6) holds.

If $m=4$ and $k=1, Q=T_{1} X_{2}^{-1} X_{1} X_{2} T_{1}$ and $P=X_{2} T_{1} X_{2}^{-1} X_{1} X_{2} T_{1} X_{2}^{-1}$, a conjugate of $Q$. Now $T_{1}^{2} \neq 1$, so $Q$ is not in a conjugate of a free factor of $G$, but since $Q$ is conjugate to $P^{-1}, Q$ is conjugate to $Q^{-1}$. By Lemma 3 , then, $Q=D E$ for some $D, E$ with $D^{2}=E^{2}=1$. But then $V^{2}=X_{2}^{-1} X_{1} X_{2} D E$, and (1.7) applies.

Suppose, then, that $m=6$ and $k=2$. We must have $|X|=|V|-1$ and $|P|=|Q|=2|V|+1$, so $X_{2}$ is empty and $T_{1}$ has length one. Let us write $X_{1}=C^{-1} \cdot a \cdot C$ with $C \in G$ and $a \in G_{i}$ for some $i \in I$ and $a^{2}=1$ and $T_{1}=b \in G_{j}$ for some $j \in I$ with $b^{2} \neq 1$. We then have $P=Q=$ $b \cdot C^{-1} \cdot a \cdot C \cdot b \cdot C^{-1} \cdot a \cdot C \cdot b$, so $b^{2} \cdot C^{-1} \cdot a \cdot C \cdot b \cdot C^{-1} \cdot a \cdot C$ is a fully cyclically reduced conjugate of $P$ which, like $P$, is conjugate to its inverse. There must then be a factorization $C_{1} \cdot C_{2}$ of $C$ such that one of the following 
holds:

$$
\begin{gathered}
C_{1}^{-1} a C_{1} C_{2} b^{-1} C_{2}^{-1} C_{1}^{-1} a C_{1} C_{2} b^{-2} C_{2}^{-1} \\
=b^{2} C_{2}^{-1} C_{1}^{-1} a C_{1} C_{2} b C_{2}^{-1} C_{1}^{-1} a C_{1} C_{2}, \\
C_{2} b^{-1} C_{2}^{-1} C_{1}^{-1} a C_{1} C_{2} b^{-2} C_{2}^{-1} C_{1}^{-1} a C_{1} \\
=b^{2} C_{2}^{-1} C_{1}^{-1} a C_{1} C_{2} b C_{2}^{-1} C_{1}^{-1} a C_{1} C_{2}, \\
C_{1}^{-1} a C_{1} C_{2} b^{-2} C_{2}^{-2} C_{1}^{-1} a C_{1} C_{2} b^{-1} C_{2}^{-1} \\
\quad=b^{2} C_{2}^{-1} C_{1}^{-1} a C_{1} C_{2} b C_{2}^{-1} C_{1}^{-1} a C_{1} C_{2}, \\
C_{2} b^{-2} C_{2}^{-1} C_{1}^{-1} a C_{1} C_{2} b^{-1} C_{2}^{-1} C_{1}^{-1} a C_{1} \\
=b^{2} C_{2}^{-1} C_{1}^{-1} a C_{1} C_{2} b C_{2}^{-1} C_{1}^{-1} a C_{1} C_{2} .
\end{gathered}
$$

If (1) is true, a length comparison on the fully reduced products on the two sides shows that

$$
C_{1}^{-1} a C_{1} C_{2} b^{-1} C_{2}^{-1}=b^{2} C_{2}^{-1} C_{1}^{-1} a C_{1} C_{2}
$$

and

$$
C_{1}^{-1} a C_{1} C_{2} b^{-2} C_{2}^{-1}=b C_{2}^{-1} C_{1}^{-1} a C_{1} C_{2} .
$$

The left sides of these two equations begin with the same normal form letter, so looking at the right sides we get $b^{2}=b$, a contradiction. Similarly, (2) yields

$$
C_{2} b^{-1} C_{2}^{-1} C_{1}^{-1} a C_{1}=b^{2} C_{2}^{-1} C_{1}^{-1} a C_{1} C_{2}
$$

and

$$
C_{2} b^{-2} C_{2}^{-1} C_{1}^{-1} a C_{1}=b C_{2}^{-1} C_{1}^{-1} a C_{1} C_{2},
$$

from which we get the contradiction $b^{2}=b$ if $C_{2}$ is nonempty or the equation $b^{-1}=b^{2}$ if $C_{2}$ is empty. This last possibility corresponds to (1.4). If (3) holds, we get

$$
C_{1}^{-1} a C_{1} C_{2} b^{-2} C_{2}^{-1}=b^{2} C_{2}^{-1} C_{1}^{-1} a C_{1} C_{2}
$$

and

$$
C_{1}^{-1} a C_{1} C_{2} b^{-1} C_{2}^{-1}=b C_{2}^{-1} C_{1}^{-1} a C_{1} C_{2} .
$$

As in (1), we derive the contradiction $b^{2}=b$. Finally, if (4) is true,

$$
C_{2} b^{-2} C_{2}^{-1} C_{1}^{-1} a C_{1}=b^{2} C_{2}^{-1} C_{1}^{-1} a C_{1} C_{2}
$$

and

$$
C_{2} b^{-1} C_{2}^{-1} C_{1}^{-1} a C_{1}=b C_{2}^{-1} C_{1}^{-1} a C_{1} C_{2} .
$$

This yields the contradictions $b^{-1}=b$ if $C_{2}$ is empty and $b^{2}=b$ if $C_{2}$ is nonempty.

Case (5.4). Suppose that $|X|<|V|, X=X_{1} \cdot X_{2}$ and $V=X_{2} \cdot X_{1}^{-1} \cdot S_{2} \cdot X_{1}$ for some $X_{1}, X_{2}, S_{2}$ with $X_{2}^{2}=1$, that $P=S_{2}\left(X_{1} X_{2} X_{1}^{-1} S_{2}\right)^{k}$ for some $k$, $0 \leq k \leq m-2$, and $Q=X_{1}^{-1} S_{2} X_{1}\left(X_{2} X_{1}^{-1} S_{2} X_{1}\right)^{m-k-2}$. 
Replacing $V$ by its fully cyclically reduced conjugate $X_{1} X_{2} X_{1}^{-1} S_{2}$ and changing notation reduces this to Case (5.3).

Case (5.5). Suppose that $|X| \leq \frac{1}{2}|V|-1, V=X^{-1} \cdot V_{2} \cdot X \cdot V_{3}$ for some $V_{2}, V_{3}$, that $P=V_{2}\left(X V_{3} X^{-1} V_{2}\right)^{k}$ for some $k, 0 \leq k \leq m-1$, and that $Q=V_{3}\left(X^{-1} V_{2} X V_{3}\right)^{m-k-1}$.

Since $|P| \leq 2|X|+3 \leq|V|+1$ and $|Q| \leq 2|X|+3 \leq|V|+1$, we have $m \leq 3$. We first consider the case that $m=2$. If $k=0, Q=V_{3} X^{-1} V_{2} X V_{3}$ conjugate to $P^{-1}=V_{2}^{-1}$ implies that $V_{3}^{2}=1$ and $V_{2}$ is conjugate to $V_{2}^{-1}$; (1.6) applies. If $k=1, P=V_{2} X V_{3} X^{-1} V_{2}$ is conjugate to $Q^{-1}=V_{3}^{-1}$, so $V_{2}^{2}=1, V_{3}$ is conjugate to $V_{3}^{-1}$, and again (1.6) applies.

Now suppose that $m=3$. In this event, we must have $|X|=\frac{1}{2}|V|-1$ and $|P|=|Q|=|V|+1$, so $\left|V_{2}\right|=\left|V_{3}\right|=1$. Let us write $V_{2}=a \in G_{i}$ for some $i \in I$ and $V_{3}=b \in G_{j}$ for some $j \in I$. Then since $Q=b\left(X^{-1} a X b\right)^{2-k}$ is conjugate to $P^{-1}=a^{-1}\left(X b^{-1} X^{-1} a^{-1}\right)^{k}$, either $a^{2}=b^{2}=1$ and $a$ is conjugate to $b$, as described in (1.3), or $a^{2} \neq 1, b^{2} \neq 1, k=1$, and there is a factorization $X_{1} \cdot X_{2}$ of $X$ such that one of the following holds:

$$
\begin{aligned}
& X_{2} b^{-1} X_{2}^{-1} X_{1}^{-1} a^{-2} X_{1}=b^{2} X_{2}^{-1} X_{1}^{-1} a X_{1} X_{2}, \\
& X_{1}^{-1} a^{-2} X_{1} X_{2} b^{-1} X_{2}^{-1}=b^{2} X_{2}^{-1} X_{1}^{-1} a X_{1} X_{2} .
\end{aligned}
$$

If (5) is true, either $X_{2}$ is empty and $a^{3}=b^{3}=1$ as in (1.5) or $X_{2}$ is nonempty and $X_{2} b^{-1}=b^{2} X_{2}^{-1}$, so that $X_{2}=b^{2} X_{3}$ and $X_{2}^{-1}=X_{3}^{-1} b^{-1}$ for some $X_{3}$, producing the contradiction $b^{2}=b$. If (6) is true, $X_{2}^{2}=1$ and

$$
X_{1}^{-1} a^{-2} X_{1} X_{2} b^{-1}=b^{2} X_{2}^{-1} X_{1}^{-1} a X_{1} .
$$

If $X_{1}$ is nonempty, $X_{1}=X_{4} b^{-1}$ and $X_{1}^{-1}=b^{2} X_{4}^{-1}$ for some $X_{4}$, whence $b^{-1}=b^{-2}$, a contradiction. Thus $X_{1}$ is empty, and $a^{-2} X_{2} b^{-1}=b^{2} X_{2}^{-1} a$ implies that $b^{-1}=a$ and $X_{2}=X_{2}^{-1}$. Thus $V=X a X a^{-1}$ with $X^{2}=1$, and (1.3) applies.

\section{REFERENCES}

1. Roger C. Lyndon and Paul E. Schupp, Combinatorial group theory, Springer-Verlag, Berlin, Heidelberg, and New York, 1977.

2. Marcel Paul Schützenberger, Sur l'equation $a^{2+n}=b^{2+m} c^{2+p}$ dans un groupe libre, C. R. Acad. Sci. Paris Sér. I Math. 248 (1959), 2435-2436.

3. Malcolm J. Wicks, Commutators in free products, J. London Math. Soc. (2) 37 (1962), 433444.

Department of Mathematics, Eastern Illinois University, Charleston, Illinois 61920 E-mail address: cflpc@eiu.edu

Department of Mathematics, Mount St. Vincent University, Halifax, Nova Scotia, CANADA B3M 2J6

E-mail address: cedmunds 01 inden.msvu.ca

Fachbereich Mathematik, Universität Dortmund, 4600 Dortmund 50, Germany E-mail address: UMA004\%DDOHRZ11. BITNETOVm.gmd.de 